\title{
DIE INTERNATIONALE WELTKARTE 1:1000000 IM ZEITGESCHEHEN
}

\author{
Von Franz Grenacher \\ Mit einer Karte
}

Im Dunkel des vergangenen Krieges ist die Gelegenheit zur Würdigung des 50jährigen Gründungsaktes der Internationalen Weltkarte (IWK.) 1:1000000, des größten Kartenwerkes, das die Menschheit je in Angriff genommen hat, unbeachtet vorbeigegangen.

Im Jahre 1891 tagte zu Bern der fünfte Internationale Geographenkongreß. Auf ihm verlas der deutsche Geograph A. PENCK eine Gedenkschrift, die darlegte, daß das Festland des Erdballs durch unzählige Forschungsreisen und Vermessungswerke nunmehr genügend bekannt sei, um zur Fertigstellung eines Kartenwerkes von internationalem Format mit einheitlichem Maßstab, Projektions- und Zeichenschlüssel schreiten zu können. Er wies auf das große Bedürfnis der Gelehrten und Ingenieure, der Wirtschaft und des Militärs hin und schlug der Fachwelt vor, sich zusammenzutun, um dieses Werk gemeinsam zu schaffen und damit den bereits bestehenden Projekten von Kartenwerken ähnlichen, aber nationalen Charakters zuvorzukommen. Er postulierte als Maßstab 1:1000000, der für zentraleuropäische Verhältnisse wohl zu klein war, dagegen für die Kolonialgebiete als das damals äußerst Mögliche galt. Zum Kartenentwurf bemerkte er, die Karte könne ihres gewaltigen Ausmaßes wegen nie zu einem Gesamtbilde gefügt werden, und aus diesem Grunde sei nur an eine polyedrische Projektion zu denken. Bei einer Gradabteilung der einzelnen Blätter von $4 \times 4$ ergäben sich etwa 880 Blätter, die Festland enthalten. Auch die Schweiz war mit einem Blatt bedacht. Weitere Vorschläge galten der Kartenzeichnung und Beschriftung. Der Kongreß stimmte den Vorschlägen zu und ernannte eine Studienkommission, in der Deutschland, England, Frankreich, Holland, Indien, Italien, Oesterreich, Portugal, Rußland, Schweden, die Schweiz, Spanien und die USA. vertreten waren. Oberst LochmanN, Chef vom eidgenössischen topographischen Büro, beauftragte als Kommissionspräsidenten Prof. E. BrüCKner und Ing.-Topograph L. Held mit der Ausarbeitung von Probeblättern, die an späteren Sitzungen vorgelegt werden konnten. Aber trotz Diskussionen an den Kongressen von 1895, 1899 und 1904 wich die Begeisterung der Ernüchterung, das nationale Prestige schwang obenauf. So schien Frankreich nicht zu begreifen, daß der Pariser Meridian nicht Ausgangsmeridian der Karte sein sollte. Die Angelsachsen wollten sich nicht dem metrischen System als Maßeinheit fügen, und Deutschland beanstandete, daß man das englische farbige Höhenschichtensystem annahm, ohne zugleich auch von Schraffen und Schummer Gebrauch zu machen. Mangels genügenden Vermessungsmaterials mußte darauf verzichtet werden.

In der Folge begannen mehrere nationale Konkurrenzwerte Gestalt anzunehmen. So verselbständigte sich die Millionenkarte Asiens des Service Géographique de l'Armée, Paris, die als eine Vorstudie des internationalen Werkes gedacht war, ebenso die Millionenkarte Afrikas der britischen Geographical Section of the General Staff (G.S.G.S.). Das Reichsamt für Landesaufnahme, Berlin, nahm eine'Millionenkarte Chinas in Arbeit, Britisch-Indien eine solche seines eigenen Subkontinents (genannt India and adjacents countries). Die Japaner laborierten im stillen an einer 1/M-Karte ganz Ostasiens, und die Russen beendigten ihre 40-Werst-Karte (1:1680031, Karte des südlichen Grenzgürtels von Russisch-Asien, praktisch eine Karte ganz Asiens). An der Verwirklichung des internationalen Werkes mußte somit gezweifelt werden. Doch siegte am Inter- 
nationalen Geographentag 1908 in Genf die bessere Einsicht, und ein besonderer Kongreß zur Aufstellung von verbindlichen Resolutionen über die Details der Karte wurde einberufen. Eine Anzahl mit Vollmachten für Sondergesandte ausgestattete Delegierte folgte einer Einladung der englischen Regierung 1909 nach London (neu kamen Australien und Kanada dazu). Dort wurden bindende Entschlüsse gefaßt über Maßstab, Entwurf, Kartensignatur, Beschriftung, Blattschnitt, Höhenschichtendarstellung und Metereinheit. Dennoch zeigt sich in der Folge, daß viele Nebensächlichkeiten das Werk an der Entwicklung hinderten. Es war zu wenig klar umrissen, wer ein bestimmtes Blatt auszuführen und welche Zentralstelle die Blätterausgabe zu überwachen habe. Dann wünschten die Engländer, Franzosen und Deutschen ihre bereits angefangenen 1/M-Kartenwerke zu vollenden, ehe sie an die neue Karte gehen wollten. Auch war für die Ausführung des südamerikanischen Kontinents nichts vorgesehen. Ein zweiter Kartenkongreß wurde nötig. Man berief ihn 1913 nach Paris. Es beteiligten sich an ihm auch verschiedene südamerikanische Staaten, so daß nun 35 verschiedene Kartenämter vertreten waren. Den Delegierten konnte ein halbes Dutzend fertige Blätter vorgewiesen werden (3 argentinische: SG 21, SH 21, SI 21, Blatt Kenhard SH 34, Boston NK 19 und Konstantinopel NK 35). Jetzt schienen sich alle Schwierigkeiten zu lösen, und es wurde beschlossen, diesem Kartenwerk amtliche internationale Geltung zu verschaffen. Der Maßstab blieb 1:1000000, Nullmeridian der von Greenwich. Als Kartenentwurf wurde eine modifizierte polykonische Projektion gewählt, die auf das Clarksche Erdsphäroid von 1880 bezogen ist. Sie befriedigte mathematisch weniger, erwies sich jedoch als praktisch. Es wurden Gradabteilungsblätter von 4 Breitengraden und 6 Längengraden gebildet. Vom Äquator ausgehend zum Nordpol erhielt die erste Blattreihe $\left(0-4^{\circ}\right)$ die Bezeichnung $A$, die zweite $\left(4-8^{\circ}\right)$ den Buchstaben $B$, die dritte $\left(8-12^{\circ}\right) \mathrm{C}$ usw. bis zum Nordpol. Das gleiche gilt für die Blattreihen vom Äquator zum Südpol. Um die Reihen der Nordhälfte von denjenigen der Südhälfte zu unterscheiden, haben jene außerdem den Vorbuchstaben $N$, die der Südhälfte S. Vom 180. Längengrad ausgehend, tragen die Blätter fortlaufend je für 6 Längengrade nebst den Buchstaben noch die Zahlen 1, 2, 3 usw. (der Greenwicher Nullmeridian trennt die Blätterreihe 30 und 31). Die Schweiz kommt so zum Beispiel gänzlich in das Blatt NL 32 zu liegen. $\mathrm{Da}$ als Blattitel wenn möglich der Name der größten oder wichtigsten darauf befindlichen Stadt gewählt wurde, empfing das die Schweiz enthaltende Blatt den Titel «Milano NL 32».

Zur Blattbezeichnung und Einteilung zurückkehrend, muß betont werden, daß durch die einfache wie geniale Einteilung, durch die die Blätter der ganzen Erdkugel ihren unzweideutigen und unverrückbaren Platz bekommen haben, die Karte eine Bedeutung erlangte, die sich erst in späteren Jahrzehnten offenbarte. Besonders zwang die IWK. die Angelsachsen, sich näher mit dem metrischen System zu befassen und trug dort so Wesentliches zur Kenntnis und teilweisen Einführung desselben bei. Je nach Wichtigkeit der Orte werden 6 verschiedene Schriftklassen für die Beschriftung verwendet. Die Darstellung der Orte selbst erfolgt in Ortsringen, und nur bei Großstädten ist der Umriß maßstäblich eingezeichnet. In der Tiefebene müssen von hundert zu hundert Meter Höhe schwarze Schichtlinien gezogen sein. Doch tragen viele Blätter auch braune Schichtlinien. Das Meer und die Seen erhalten Tiefenlinien. Ihr charakteristisches Aussehen erhält die Weltkarte jedoch erst durch die Farbtönung der Schichtstufen. Nach einer genau festgelegten Farbenskala gehen die Farben vom Sattgrün (0-100 Meter Höhe), in der 100- bis 200-Meter-Schichtstufe ins Hellgrün über; ab 200 Meter beginnt ein Hellbraun, das in verschiedenen Farbstufen im Ziegelrot in Höhen von 4000 Meter endet. Der ewige Schnee bleibt weiß. Ebenso wechselt die Meeresfarbe vom Hellblau für seichte Gewässer bis zum Dunkelblau der Tiefsee. Diese Darstellungsart wird kurz layered System genannt. Um nationale Empfindlichkeiten zu schonen, mußten genaue Vorschriften für die Beschriftung aufgestellt werden. 
Natürlich wurde als Schrift das lateinische Alphabet adoptiert. Ferner forderte man, daß die Orts- und anderen geographischen Namen in der offiziellen Sprache des Landes, dem sie zugehören, einzutragen seien. Zum Beispiel war im Blatt München NM 32, das Deutschland 1925 herausgab, Straßburg nicht französisch als Strasbourg eingetragen, und deshalb konnte das Zentralbüro das Blatt nicht offiziell anerkennen.)

Nur langsam nahm die Karte Gestalt an. Als 1914 der erste Weltkrieg ausbrach, mögen etwa 10 verschiedene Blätter bestanden haben. Wiederum lag Grund vor, anzunehmen, das Werk werde daran zerschellen. Da erkannte der britische G.S.G.S. deren hohen Wert als Operationskarte für diverse militärische Zwecke und begann insgeheim und auf eigene Faust (natürlich ohne Wert auf offizielle Anerkennung durch das Zentralbüro zu legen) ein ganzes Netz von Blättern über Europa und Südwestasien auszubreiten. Anfänglich ließ er eine Serie Europablätter ohne layered System von A. R. HrNks in der Royal Geographical Society zusammenstellen. Doch später übernahm es der G.S.G.S. in ejgener Regie, dieses Werk im layered System zu veröffentlichen. Mit großer Regelmäßigkeit und Meisterschaft erschien Blatt um Blatt. 1915 waren es 7, 1918 schon 68, so daß anläßlich der Freigabe für die Oeffentlichkeit die ganze interessierte Welt überrascht war.

In Brasilien, das 1922 seine Hundertjahrfeier mit einer Weltausstellung verband, zeichnete der dortige Ingenieurverband mit Feuereifer an den 45 Blättern des Landes. Zur gleichen Zeit begann unter Leitung von I. BowmanN, einem Freund Wilsons, die American Geographical Society of New York (kurz A.G.S.) in größeren Zeitabständen die 107 Blätter ihrer Millionenkarte des gesamten Lateinamerikas von Kalifornien bis Feuerland zu publizieren. So entstand ein Werk von großer Eindrücklichkeit. Doch mußte die Herausgabe der letzten Blätter des erneuten Kriegsausbruchs wegen bis 1945 zurückgestellt werden. Vor Jahresfrist konnte nun die Herausgabe des Werkes gefeiert werden. Seine Herstellungskosten beliefen sich auf rund eine Million Schweizer Franken. 1925 begann der Service Géographique de l'Armée, Paris, das sogenannte Croquis de l'Afrique Française 1/M im Blattschnitt und Entwurf der IWK. herauszugeben, mußte aber wohl in Ermangelung genügender Messungsunterlagen auf die layered Schichten und einige weitere Details verzichten. Aber die Fachwelt verfügte 1939 über ein weiteres einheitliches Kartenwerk von 52 Blättern von Gibraltar bis zur Kongomündung. In den Jahren 1920 bis 1939 publizierten des weitern: Indien 21 Blätter, Japan 14 Blätter, Australien 9 Blätter, die Topograph. Inrichting in Weltevreden (Batavia) 11 Blätter, Ägypten 6, Indochina 7, Siam 7, Minist. d. Colonies Portugal von Mozambique 3, Kanada 3, Angloägyptischer Sudan 12, Tschechoslowakei 1, Polen 4, Finnland 4, Frankreich (Stammland) 4, Deutschland 5, Britische Inseln 6, Ungarn 1, Neuseeland 3, Norwegen 4, Portugal 2, Spanien 4, Südwestafrika 1, Schweden 4 Blätter. Italien schuf außer den 5 Blättern seines Mutterlandes beim Ausbruch des Abessinienkrieges 1935 die Carta delle Africa Orientale Italiana in 8 Blättern und 1939 die 5 Blätter Libyens. Der letzte offizielle Bericht des Zentralbüros im Jahre 1938 verzeichnet 32 verschiedene Herausgeber und etwa 375 erschienene Blätter, wobei allerdings viele Doppel waren.

Die USA. begnügten sich mit der Herausgabe von 4 Blättern, die, obwohl technisch hervorragend, für sie nur Versuche waren. Ihr Prestige verlangte, daß keine provisorischen Blätter erschienen, bevor der Stand der Vermessungen so weit abgeschlossen war, daß sich diè Publikation definitiver Blätter rechtfertigte.

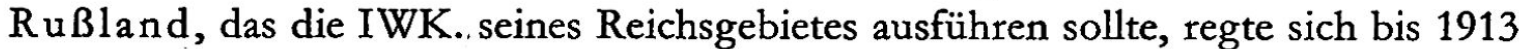
kaum; die Lateinschrift und das Metermaß dürften es abgehalten haben. Erst die Sowjetregierung entschloß sich seit etwa 1920 offiziell, an Stelle ihres alten Werstmaßes das metrische System zu adoptieren. 1929 meldete das russische Militärwochenblatt, daß mit dem Druck einer neuen Karte $1: 1 / \mathrm{M}$ (24Werst) in sieben Farben begonnen worden, sei. Der Grundriß war schwarz, die Wege erschienen rot, Höhendarstellung in Schicht- 
linien und Schummerung. Die Karte wurde wie die ganze übrige russische Kartographie streng geheimgehalten. Trotzdem gelangten einige Blätter nach Westeuropa. Aus ihnen war zu ersehen, daß dieses Kartenwerk in Entwurf, Blattschnitt und Blattbezeichnung auf der IWK. basierte. Geheimhaltung und zyrillische Beschriftung hinderten naturgemäß die Anerkennung durch das Zentralbüro. Bis 1939 waren ungefähr 60 Blätter fertig (hauptsächlich Europäisch-Rußland und die Region der transsibirischen Eisenbahn).

Das Vorhandensein eines dominierenden Kartenwerkes zeitigte aber Auswirkungen auf verschiedene Kulturgebiete. Die Archäologen, die ihre verschiedenen Funde von römischen Orten, Gräbern, Befestigungen, Straßenstücken usw. auf einer Generalkarte zusammenfassen wollten, bedienten sich der IWK., um ihre "Tabula Imperii Romani» herauszugeben. Bis heute sind 2 englische, 4 italienische, 1 französische und 4 ägyptische Blätter erschienen. Auch die Internationale Luftfahrkommission erkannte ihre großen Vorteile für die Aviatik und gebrauchte sie als Grundlage für die Internationale Luftfahrtkarte $1: 1 / \mathrm{M}$. Die hauptsächliche Änderung ist die Weglassung der grünen Schichtfarbe für das Tiefland. Englische, französische, polnische und 1 griechisches Blatt zeugen für die Entwicklung dieser Spezialkarte.

Beinahe zwei Drittel der IWK. fallen auf Meeresgebiet, für das die Konferenzen bisher keine Ausführungsvorschriften erließen, obwohl Fürst Albert von Monaco, dessen Bureau Hydrographique für die Internationale Meereskarte als Zentralstelle zuständig ist, sich anerboten hatte, versuchsweise einige Meeresblätter zu bearbeiten. Er scheint keine Zustimmung erhalten zu haben. Daher publizierte TH. Stocks, vom Institut für Meereskunde, Berlin, 1936 auf eigene Faust ein erstes Versuchsblatt, SO 26 Südsandwich-Inseln, dem noch weitere folgen sollten. Der Weltkrieg unterbrach jedoch das Vorhaben. Viele Echolotungen ergeben ein plastisches Bild vom Meeresboden; indessen konnte von einer eigentlichen Topographie noch nicht gesprochen werden.

Noch maßgebender war der Einfluß der Weltkarte auf die übrige Kartographie. Blatteinteilung, Schnitt und Bezifferung, die den ganzen Globus umfassen, erlangten nun auch für viele andere Kartenwerke Gültigkeit. So begann Frankreich die mittleren Maßstäbe 1:500000, in einigen Fällen sogar 1:50000 als Unterteilungen der Weltkarte zu konstruieren. Desgleichen führten die G.S.G.S. in Southampton und der India Survey, Calcutta, für ihre Kartenwerke des vorderen Orients im Maßstab $1: 250000$ und 1:253440 (von Smyrna bis Rangoon reichend) eine Einteilung und teilweise auch eine Bezifferung ein, die diese als Untergruppen der Weltkarte zu betrachten gestatten. Ebenso gilt dies für englische Kartenwerke in Uganda und Britisch-Westafrika. Ferner paßte der G. S. G. auch Kartenwerke im Maßstab 1: 2000000 von Afrika und 1: 4000000 von Asien der IWK. an. Bei der ersteren sind immer 4 anschließende Weltkartenblätter auf einem Kartenblatt der 1:2000000-Karte mit entsprechender Bezeichnung und 25 Weltkartenblätter auf der $1: 4000000-K a r t e ~ z u s a m m e n g e f a ß t$. Weiter ist Mexikos offizielle $1: 500000$-Karte eine Unterteilung der IWK., ebenso die in den USA. publizierte Karte 1:250000 von Mittelamerika. Auch bei Kanadas 1:506880-Karte hat die Einteilung noch leichten Bezug auf die IWK. Der U.S.A. Coast and Geodetic Survey in Washington gibt eine hervorragende 1:500000-«Sectional»-Flugkarte im farbigen Höhenschichtensystem heraus. Ein solches Blatt stellt immer die nördliche bzw. südliche Hälfte eines Weltkartenblattes dar. Das 1: 500000-Kartenwerk des Geological Survey in Washington, das die einzelnen USA.-Staaten separat darstellt, nennt sich ausdrücklich «Basis Map» zur IWK. Ein weiteres zusätzliches Kartenwerk in Unterteilung und mit Blattbezeichnung der WK. im Maßstab 1:500000 begann West- und Zentraleuropa zu überziehen. Frankreich, Polen und später Deutschland bearbeiteten je ihre eigenen Territorien. Für Frankreich wurde diese Karte zur nationalen Landesflugkarte, für Polen dessen offizielle 1:500000-Karte und für Deutschland die Flug- 
karte $1: 500000$. Letztere wurde im Staatsauftrag vom bekannten Verlag Justus Perthes in Gotha aus der ehemaligen "Vogelschen Karte» abgewandelt.

Erwartungsgemäß sollten die strengen Vorschriften des Zentralbüros in Southampton ein Kartenwerk von großer Einheitlichkeit zeitigen. Aber nur wenige Dutzend Blätter erfüllten alle Vorschriften und wurden offiziell anerkannt. Um deshalb nicht allzu eingeschränkt zu bleiben, sah sich das Zentralbüro veranlaßt, eine Mehrzahl von Blättern anzuerkennen, die in irgendeiner Art nicht allen Vorschriften gerecht werden.

Naturgemäß tragen auch die vermessungstechnischen Grundlagen zu großen Unterschieden bei. Die zentraleuropäischen Blätter konnten auf Grund erstklassiger Vermessungsergebnisse generalisiert werden. Im Innern von Südamerika oder der Sahara usw. dagegen mußte ein unsicheres Netz von Routenitineraren von Forschungsreisenden zur Herstellung genügen. Aus diesem Grund wurde zuerst von der A.G.S. für ihre Karten ein Verläßlichkeitsdiagramm beigegeben, aus dem die Meßmethode in jedem Kartenteil ersichtlich ist. Von einer Kartenausgabe zur anderen kann man oft in solchen Gegenden Korrekturen von seitlich verschobenen Flußläufen und anderen Details von Dutzenden von Kilometern ablesen. Natürlich sind solche Blätter nur als provisorische Ausgaben zu bewerten. In den dreißiger Jahren erschienen erstmals ganze Blätter auf Grund luftphotogrammetrischer Aufnahmen, wie zum Beispiel die 4 Blätter von Holländisch-Borneo. Viele Nationen legen Wert auf die Darstellung typischer Eigenheiten.

Es darf gesagt werden, daß die Blätter des englischen Mutterlandes vom Ordnance Survey, Southampton, jene Norwegens, Deutschlands, Australiens Blatt SI 55 Canberra, die USA.-Blätter NJ 10 San Francisco und NK 18 New York, das polnische Blatt Wruno NN 35 sowie viele Blätter der A. G.S., wie NC 19 Caracas, zu den Spitzenblättern zu zählen sind. Daneben gibt es aber Blätter primitivster Anfängertechnik.

Mit dem Ausbruch des zweiten Weltkrieges wurde der Vertrieb der verschiedenen Blätter durch die kriegführenden Staaten verboten und die Auflagen dem Militär reserviert. Auch die Publizität über sie war unterbunden, und erst seit Kriegsende entschleiert sich nun ihr weiteres Schicksal. Schon im September 1940, anläßlich der Bombardierung von Warschau, wurde das polnische Militärgeographische Institut durch Volltreffer zerstört und mit ihm alle seine Kartenvorräte. Der französische Service Géographique de l'Armée mußte sich unter deutscher Besetzung in ein Institut National de Cartographie umbenennen und konnte nur einige wenige Saharablätter der Weltkarte erneuern. Justus Perthes in Gotha begann im Maßstab, Zeichen und Schnitt der Weltkarte eine sogenannte Sven-Hedin-Karte Innerasiens zu konstruieren; geplant waren 50 Blätter, aber nur 3 erschienen. Die Kriegsverhältnisse verhinderten ein weiteres Gedeihen. Das Heim des Zentralbüros der "Carte du Monde» sowie dasjenige des G.S.G.S. in Southampton fielen ebenfalls einem feindlichen Angriff aus der Luft zum Opfer, samt Kartendepot und Platten. Da an eine schnelle Herstellung nicht zu denken und der Kriegsbedarf ungeheuer war, verfielen die Engländer auf den Ausweg, die Karten durch eine Ausgabe auf photolithographischem Wege zu ersetzen. Diese neue Serie wird Ground/Air-Typ genannt. Auf dünnerem, zähem Papier wird die vergröberte Konturenzeichnung gedruckt; Meer und Gewässer sind blau, die Meeresisobathen weggelassen. Die 0-100-Meter-Höhenschicht bleibt weiß, die von 100 bis 200 Meter bekommt einen feinen violetten Raster, die Höhen über 200 Meter haben einen Braundruck, vom feinsten Raster bis ins Vollbraun gehend in über 5000 Meter Höhe. Schöne Beispiele dieses Typs sind die Blätter NI 30 Fez, NK 37 Batumi und NB 48 Trengganu; dieser Typ wurde jedoch bald fallen gelassen und durch den Army/Air Style ersetzt. Die Grünplatte des Tieflandes ist hier ebenfalls weggelassen. Die Höhen über 200 Meter werden nur noch mit einem Violettdruck behandelt, ausgehend vom feinsten Raster bis Vollviolett in über 5000 Meter. Verschiedene Blätter erhielten eine weitere rote Platte für die aeronautischen Bedürfnisse. Eigentümlicherweise wurde für 


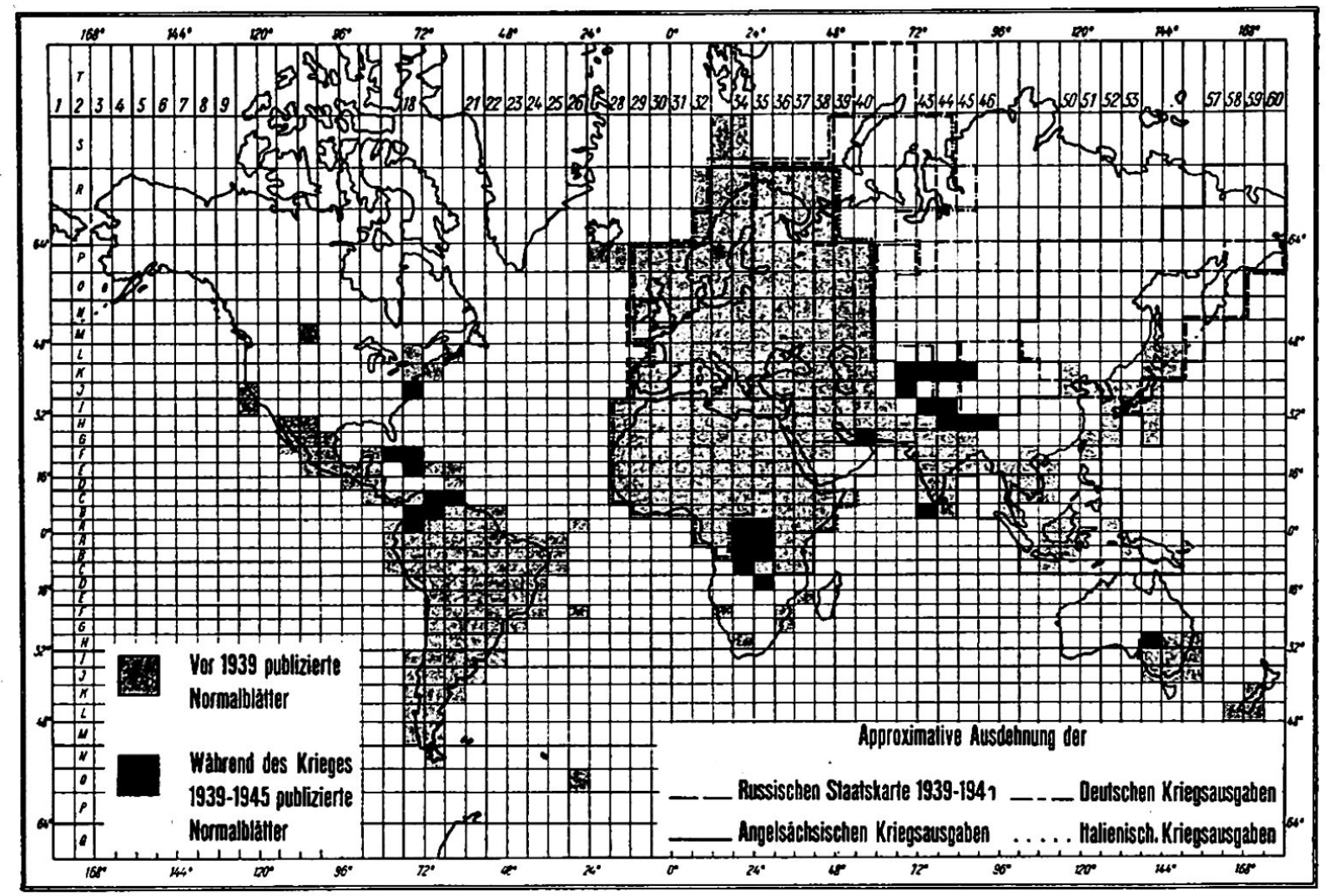

Die Internationale Weltkarte 1:1000000 im Weltkrieg 1939-1945

(Die Angaben über die Russische Staatskarte gelten nur von 1939-41)

West- und Zentraleuropa auch der Blattschnitt der Weltkarte nicht mehr eingehalten; ein Sammelsurium von verschieden großen Blättern (8 auch 9 Längengrade) ersetzte die Weltkarte. Doch vom 30. Längengrade an nach Osten zieht sich im alten Weltkartenschnitt ein ununterbrochenes Netz von Blättern bis nach China und Neuguinea. Hier kann diese Karte nicht als Ersatz der alten gewertet werden, da viele bisher unpublizierte dazustoßen, wie Samarkand, Manasarowar, Mandalay, Hankau und Mukden. Die Blätter Ostafrikas wurden durch einen schönen farbigen "Security Typ» ersetzt, der jedoch in der Farbgebung nicht den Satzungen der Weltkarte entspricht. Die benötigten Blätter der Sahara wurden ohne Veränderung vom französischen Service kopiert. Gänzlich überraschend und neu ist eine Serie Kartenblätter, G.S.G.S. Edition and Edition A.M.S. genannt, die sich von der Beringstraße über Ostsibirien, Sachalin, Japan, Philippinen und Celebes hinzieht, an der zum erstenmal zwei Herausgeber: die englische Geographical Section of the General Staff, Southampton, und der Chief of Engineers, U. S. Army Map Service, Washington, beteiligt sind und in Eintracht zusammenarbeiten. Bei diesen Blättern ist wieder das alte layered System der Weltkarte im Schnelldruckverfahren angewendet worden. Ein gutes Beispiel ist das Blatt NK 52 Wladiwostok, auf dem 10 Farben verwendet wurden. Bedauerlicherweise muß als weiterer Verstoß gegen das Weltkartenwerk vermerkt werden, $\mathrm{da} ß$ auf den Blättern der Philippinen die Höhenschichtlinien in Fußmaßen und die Meerestiefen in Faden dargestellt sind. Was die G.S.G.S./A.M.S.-Serie anbetrifft, so wird sie in Washington hergestellt; zusammen mit den in England produzierten Army/ Air-Style-Blättern ergeben sich über 90 vollkommen neue Weltkartenblätter. Über den Verbrauch dieser Kriegsausgaben können wir uns ein gutes Bild machen. Von der zweiten Army/Air-Edition des Blattes NG 42 Sind (1945), worauf auch Karachi mit seinen 9 wichtigen Fluglandeplätzen figuriert, wurden zum Beispiel 40000 Kopien gemacht. Der G.S.G.S. hat des weitern die von den Franzosen, Deutschen und Polen 
angefangenen 1:500000-Kartenwerke (Unterteilungen der IWK.) zu einer Air Map 1: 500000 umgestaltet und sie auf beinahe ganz Europa und Nordafrika ausgedehnt. Ja, er hat sogar noch eine weitere Unterteilung im Maßstab 1: 250000, die Air-MapSerie, für West- und Zentraleuropa eingeführt. Der India Survey hat in der Kriegsperiode einen großen Teil seiner Weltkartenblätter erneuert und etwa 8 weitere neue Blätter im layered System ausgeführt.

Ein weiteres Beispiel von Zusammenarbeit bezeugen die Blätter des belgischen Kongo. Die Vermessungsorgane des Service Cartographique du Ministère des Colonies, die während des Krieges abgetrennt von der Heimat in Kongo verblieben, wären außerstande gewesen, ihre Originalzeichnungen zu vervielfältigen. Da stellte Südafrika seine militärische Kartendruckerei zur Verfügung, wodurch die Alliierten aus den 13 neuen Blättern ihren Nutzen ziehen konnten.

Als einziges regulär anerkanntes Blatt des Geological Survey in Washington erschien im Krieg vom Gebiet der USA. das Blatt Chesapeake Bay NJ 18 (es handelt sich eigentlich um Blatt Washington). Dieses Blatt verfügt zum erstenmal auch über eine genaue Meeresbodentopographie. Vor und während des Krieges haben die Amerikaner eine sogenannte Radio Acoustic Ranging, (RAR)-Aufnahmen-Methode, entwickelt, mit der nun der Meeresboden in einwandfreier Weise kartiert werden kann. Plastisch eindrücklich sind der Verlauf des Umrisses der Kontinentalscholle und der Abbruch zur Tiefsee dargestellt. Das Blatt ist verheißungsvoll für die zukünftigen reinen Ozeanblätter, deren Ausführungsmöglichkeit bisher immer noch in Zweifel gezogen wurde.

Seit 1940 entfaltet die USA. für die Kartenherstellung ungeheure Kräfte; ein rundes Dutzend verschiedener kartenproduzierender Organisationen und Institute mit aber Tausenden von Mitarbeitern hatten sich den Kriegsbedürfnissen unterzustellen. Es war daher anzunehmen, daß außer der im Verein mit dem englischen G.S.G.S. publizierten Weltkartenserie noch weitere Werke (im Zusammenhang mit der IWK.) geschaffen worden sind. Nun erweist sich, daß der Army Map Service sich auch der Basis Map zur Weltkarte, d.h. der 1:500000-Karte, deren immer 4 auf ein Weltkartenblatt entfallen, angenommen hat. Diese erstreckt sich über große Gebiete. Die ganze Küstenzone Grönlands wurde damit bedacht, ebenso ganz Südamerika; doch steckt dort die Arbeit noch in den Anfängen. Für Melanesien existiert sie längs eines Streifens von New Irland bis zur Insel Erromango (17 BL). Sonderbarerweise wurde für eine Kartenserie 1:500000 von Westchina (30 Blatt) der Blattschnitt leicht abgeändert, so daß sie kaum mehr mit der Weltkarte in Verbindung steht. Endlich wurde unter dem Siegel des Kriegsgeheimnisses und der Oberleitung des Aeronautical Chart Service der Army Air Forces ein Millionenkartenwerk in Hunderten von Blättern über den ganzen Erdball gezogen, sozusagen auf einen Anhieb und in einem Guß. Wenn das Werk auch Anzeichen der Hastigkeit in der Ausführung zeigt, grenzt diese Leistung doch ans Ungeheuerliche. Bedauerlicherweise erinnert keine Bezeichnung mehr an die IWK.; aber dennoch ist leicht ersichtlich, daß der "World A erona utical Chart» der IWK. zu Gevatter gestanden hat. (Luft- und Seekarten, die Navigationszwecken dienen, werden englisch nicht als Maps, sondern Charts bezeichnet.) Daran erinnert zunächst ein ähnliches layered System, auch wenn es in Fußmaßen eingetragen und die Zeichnung gegenüber der Weltkarte mehr generalisiert ist. Aus Zweckmäßigkeitsgründen wurde die Blatteinteilung leicht abgeändert. Die Blatthöhe, die ebenfalls 4 Breitengrade beträgt, ist dieselbe wie bei der Weltkarte. Die Blattbreite von 6 Längengraden, die bei der Weltkarte gegen Nordpol und Südpol der zusammenlaufenden Meridiane wegen immer kleiner wird, wurde bei der World Air Chart vom Äquator aus in der neunten Blattreihe auf 7 Längengrade, in der elften Reihe auf 8 und in der dreizehnten Reihe auf 9 Längengrade usw. ausgedehnt. In Wirklichkeit bleiben so alle Blätter gleich groß $(21 \times 28$ Zoll). Diese Normung bedeutet einen großen Vorteil für den Kartentisch eines 
Flugzeuges, ebenso für dessen Navigation, und dieses Werk ist ja vor allen Dingen für das Flugzeug geschaffen worden. Neu ist auch, daß dieser Kartentyp sich nicht mit einer einzigen Projektion begnügt. Er benötigt gleich deren drei, die vom Äquator zonenweise nach den Polen hin wechseln. Naturgemäß wird großer Wert auf aeronautische.Eintragungen gelegt. Wie eine Anmerkung besagt, ist die Karte chemisch präpariert, so daß sie auch bei Nacht, bei allen Beleuchtungsarbeiten gleich gut gelesen werden kann. Sie hat der IWK. gegenüber den großen Vorteil, auch für Sibirien, Zentralasien, Nordamerika, Südafrika, Australien und Ozeanien greifbar zu sein, für welche Gebiete es noch gar keine Weltkartenblätter gibt!

Trotz dem eisernen Vorhang, hinter dem die Sowjetunion ihre gesamte Kartographie verbarg, sind wir heute durch die aufschlußreiche Publikation eines Deutschen, W. Krallert (1943), zur Hauptsache über diese aufgeklärt worden. An Hand von erbeuteten russischen Karten und Schriften konnte er feststellen, daß der Rußland betreffende Teil der IWK. (wahrscheinlich in einer dritten Neubearbeitung) in der Sowjetunion zur Staatskarte par excellence geworden ist, nach der sich die ganze übrige topographische Kartographie auszurichten hat. Diese Staatskarte legt sich heute über den ganzen eurasischen Kontinent. Gleich zwei Ausgaben, eine im layered System und eine uneingefärbte mit grüner Waldsignaturenplatte, existieren nebeneinander. Für die Konstruktion eines jeden Blattes wurde zuerst ein Vorschriftenheft mit den Anleitungen verfaßt. Als Entwurf wurde die polyedrische Projektion gewählt. Als Blattbezeichnungen werden die lateinischen Buchstaben und gleiche Bezifferung wie bei der Weltkarte beibehalten, sonst ist aber die ganze Beschriftung zyrillisch. Die topographischen Kartenwerke der ganzen Sowjetunion in den größeren Maßstäben $1: 500000,1: 200000,1: 100000$ u. a. sind als Unterteilung der Staatskarte 1/M konstruiert. So sind 4 Blätter der 500000 er-Karte ein Normal-Staatskartenblatt, 36 Unterteilungen ergeben den Blattschnitt der 200000er-Karte und 144 Unterteilungen den Schnitt zur 100000er-Karte usw. Auch der geologischen Übersichtskarte 1:1000000 in 220 Blättern scheint die Einteilung der IWK. zugrunde zu liegen. In der sowjetrussischen Staatskarte ist der IWK. also indirekt die größte Anerkennung gezollt worden!

Die Achsenmächte verfügten am Kriegsvorabend nur über wenige eigene Blätter der IWK. Doch in Anbetracht der Wichtigkeit des Kartenwerkes begannen sie, die ihnen für Kriegsoperationen wesentlich erscheinenden Zonen den bereitgestellten Kartensammlungen zu entnehmen, um sie für die «Edizione speciale fuori commercio per exclusivo uso militare» und für die «Sonderausgabe nur für den Dienstgebrauch» zu kopieren - eine Praktik, der sämtliche kriegführenden Staaten gehuldigt haben. Diese Kopien sind zum Teil hervorragend gelungen. Teilweise wurden dabei bisher uneingefärbte Blätter mit dem layered System versehen, andere verbessert, erneuert oder direkt neu gezeichnet. Italien verfügte so schließlich über ein Weltkartenwerk, das vom Nordkap über den Ural und die Levante nach Abessinien und Tunis reichte. Der deutsche "Generalstab des Heeres, die Abteilung für Kriegskartographie und Vermessungswesen II» ging noch gründlicher zu Werke. In glänzenden Nachdrucken wurden 1940 ganz Europa, Afrika bis zum Kongo, Arabien und Persien, Teile Indiens, die Pamire und ganz Europäisch-Rußland dargestellt. Die Deutschen genügten sich aber nicht mit dem Kopieren; viele Blätter des Sudans und Arabiens wurden mit dem layered System versehen; auf verschiedenen Sahara-Blättern wurde das Pisten- und Karawanennetz neu revidiert und in rotem Überdruck eingetragen. Einige Blätter Afghanistans und Indiens (Kabul, Kaschmir und Jarkand), die es bis dahin noch gar nicht gab, wurden an Hand des "India and adjacent countries»-Kartenwerkes neu gezeichnet. Zu Kriegsanfang mußte der Generalstab mit der einzigen greifbaren Quelle für Europäisch-Rußland, mit der 1916 bis 1920 publizierten unlayered Serie des englischen G.S.G.S., vorliebnehmen. Er versah sie jedoch sofort für seine Zwecke mit dem 
layered System. Einzelne wichtige Blätter, wie NN 36 Smolensk, NM 36 Kiew, NL 36 Odessa, NK 37 Batum und NK 38 Tiflis, wurden sogar vollständig neu gezeichnet. Nach Exbeutung großer russischer Kartenbestände wurde 1942/43 eine weitere Ausgabe angefertigt, die dem russischen Material entnommen ist. Natürlich wurde die russische Beschriftung ins Latein umgesetzt. Viele Blätter der Staatskarte 1/M wurden auf diese Art umgadruckt, und mit großem Geschick und ohne zu stören die Grünplatte der Waldsignatur (übrigens ein für Rußland typischer, unerläßlicher Kartenbestandteil) mitverwendet; ebenso die Blätter der 500000er-Karte. Auf vielen Blättern wurde noch ein neuartiges Fliegergitter aufgedruckt. Diese erweiterte Auflage im beschriebenen Typ erstreckte sich nun von Blatt NM 34 Wien bis zum. Nordkap, zur Obmündung, Sverdlowsk, zum Balkasch-See und Pamirgebirge. Das Werk entwirft für Rußland ein völlig neues, tiefer in topographische Einzelheiten gehendes Bild, das der Geographie weitere Kenntnisse vermittelt und Irrtümer korrigiert. Grenzen von Naturschutzpärken sind zum Beispiel eingetragen, bei Archangelsk sogar die Gestelle der Wälder. Blatt Kuijbischew NN 39 zeigt die Umrisse des riesigen Wolga-Stauprojektes, Blatt Iwanowo NO 37 den neuen Rybkiner Stausee (etwa dreimal Genfersee), der der Speisung der Schiffahrtskanäle zwischen Moskau bzw. Wolga und der Ostsee dient. Mit dieser Kartenserie verdient Deutschland Anerkennung für den hervorragenden Umdruck; aber auch die Russen erweisen sich als erstklassige Kartographen, soweit sich das ursprüngliche Kartenmaterial beurteilen läßt. Wenn die Schweiz darauf: stolz ist, in der kartographischen Darstellung der Gebirgswelt an der Spitze zu stehen, so gebührt den Russen die Anerkennung, in der Darstellung der Tiefebene Meister zu sein. Dies ist ein nicht gering zu wertender Faktor, bedeutet doch das Tiefland die Besiedlungszone von $90 \%$ der Menschheit.

Abschließend sei der verschiedenen Blätter gedacht, die die Schweiz darstellen. Ihrer Kleinheit wegen konnte dieser, wie vermerkt, kein offizielles Blatt zur Bearbeitung und Herausgabe zugeteilt werden. Dagegen haben sich bis heute eine ganze Anzahl verschiedener ausländischer Herausgeber mit diesem Blatte beschäftigt. So erschien 1915 Blatt NL 32 Milano ohne Höhenschichteneinfärbung, von der Royal Geographical Society in London bearbeitet. 1926 publizierte der G.S.G.S. dasselbe, nun schön farbengetönte Blatt im layered System. 1935 erschien eine weitere Auflage mit Nachträgen. 1927 wurde das offiziell anerkannte Blatt NL 32 Milano der «Carte internationale du monde au millionième» vom Istituto Geografico Militare, Firenze, herausgegeben. Es kann unsere verwöhnten Ansprüche kaum befriedigen. Nebst Fehlschreibungen, wie «Wierzwaldstättersee», und Fehldarstellungen, wie "Wollerau als wichtigster Ort am linken Zürichsee Ufer», ist die Farbtönung des Hochgebirges im layered System schwächlich ausgefallen. Um 1928 erschien Blatt Mulhouse NL 32 der Carte de France 1/M, einer nationalen Ausgabe der IWK.; doch ist nur das französische Territorium bearbeitet. $1932 \mathrm{kam}$ das Blatt Milano NL 32 der "Tabula Imperii Romani» des gleichen Istituto Geografico Militare heraus. Es scheint sehr selten zu sein und ist seit langem vergriffen. 1935 erschien ein Halbblatt NL 32 ("Westliches Alpengebiet») des Reichsamtes für Landesaufnahme, Berlin. Es zeigt nur die nördliche Hälfte eines Normalblattes, zur Hauptsache die Schweiz und Westtirol. Technisch ist es einwandfrei; doch verzeichnet es einen nicht existierenden See bei Büren an der Aare. Von ihm war auch eine Abart, «morphologische Spezialausgabe» genannt, käuflich. Es fehlte ihm die Schwarzplatte, so da $B$ Namen, Eisenbahnen und Ortschaften weggefallen sind. 1936 erschien Blatt NL 32 Milano der "Carte Internationale Normale Aéronautique», ebenfalls durch das Istituto Geografico Militare, Firenze, publiziert. Die grüne Farbplatte für das Tiefland fehlt, dafür sind in einer weiteren violetten Platte die aeronautischen Belange eingetragen. Um 1940 erschien Blatt Milano NL 32 des deutschen Generalstabs des Heeres, Abteilung für Kriegskartographie und Vermessungswesen II. Es handelt sich um eine nur leicht korrigierte Kopie der offiziellen italienischen Aus- 
gabe von 1927. Etwa um die gleiche Zeit druckte der russische Generalnyj Schtab RKKA., Moskau, das Blatt NL 32 als Ergänzung zur Jewropejskaya Tschast SSSR. $1: 1000000$. Sodann erschien um 1942 Blatt L 32 und Pt. L 31 «Lyon/Milano» (was NL 32 and Parts of NL 31 bedeutet. Es reicht vom dritten bis zwölften Grad östlich Greenwich) der "Army/Air Edition» des englischen G.S. G.S. Im Vergleich zum Normalblatt ist es im Westen um 3 Grade erweitert; es trägt als Höhenschichtenfarben ab 200 Meter die früher beschriebenen violetten Stufen und weicht somit merkbar vom offiziellen Typ ab. Im folgenden Jahr erschien Blatt 253 der World Aeronautical Chart, «Lake of Geneva» (nach Gepflogenheiten des G. S. G. S. für nicht blattschnittgetreue WK.Blätter hätte es mit Pt. NL 31 und Pt. NL 32 bezeichnet werden können). Herausgeber ist der Aeronautical Chart Service der Army/Air Forces, Washington. Ausführende kartographische Anstalt: Coast and Geodetic Survey, Washington (in Lamberts Conformal-Konischer Projektion). Die Blattbreite geht vom 3. bis 11. Längengrad. Es besitzt Sechsfarbendruck. Das angewandte layered System zeigt von 0 bis $1000 \mathrm{Fu}$ Höhe Sattgrün, von 1000 bis 2000 Fuß Hellgrün, ab 2000 Meter ein Braun, das in 6 Farbenstufen bis auf $15000 \mathrm{Fu}$ angewendet wird. Es ist sauber gearbeitet; doch sind die Angaben im Eisenbahn- und Straßennetz fehlerhaft. Es weicht wesentlich vom IWK.Typ ab.

Nachdem die übergroße Mehrzahl der publizierten Blätter, die in irgendeiner Weise der IWK. angehören, offiziell nicht anerkannt ist, wäre es für die schweizerische Kartographie eine Ehrensache, ein Blatt NL 32, das einer landeseigenen Offizin entstammt, zu schaffen und damit, wenn auch nicht quantitativ, so doch qualitativ an der weitərn Entwicklung der IWK. teilzunehmen, der offenbar trotz zahlreichen Hemmnissen unzweifelhaft die Zukunft gehört.

\section{LITERATUR}

1. Anonymus: Resolutions and Proceedings of the International Map Commitee, assembled in London, November 1909. Official International Commitee, London 1910.

2. - Die Weltkartenkonferenz vom November 1909. Petermanns Mitteilungen 57, I, 1911, 29-31, 88-89.

3. - Catalogue of maps, G.S.G.S. War Office, London 1930, 1935 und 1946.

4. - The international 1:1000000 map. Report for 1938. Southampton 1938.

5. - Catalogue of maps. Army Map Service. Washington (o. J.).

6. - Indexblätter der World Aeronautical Chart. Headquarter, Aeronautical Chart Service, Washington 1946.

7. - The map of Hispanic America on the scale of $1: 1000000$. Geographical Review, 36, 1946,1-28.

8. BrüCKNER, E.: Bericht über das Projekt einer Erdkarte im Maßstab 1:1000000. Jahresbericht der Geographischen Gesellschaft in Bern, 11, 1891/92, 290-299.

9. - Die Internationale Weltkartenkonferenz in London. Mitteil. der Geogr. Gesellsch., Wien 1910, 217-229.

10. - Die Weltkartenkonferenz in Paris im Dezember 1913. Mitteil. derGeogr. Gesellschaft, Wien 1914, $211-224$.

11. Finsterwalder, R., und Hueber, E.: Vermessungswesen und Kartographie in Afrika. Afrikanisches Handbuch der Kolonialwissenschaften. Berlin 1943, Bd. I.

12. KosACK, H. P.: Die Fortschritte der Kartographie des Auslandes. Jahrb. d. Kartographie I, 1941, $238-248$.

13. Krallert, W.: Die Planmäßigkeit auf dem Gebiet sowjetrussischer kartographischer Arbeiten. Archiv für Landes- und Volksforschung 7, 1943, 12-44.

14. MEYFR, H.: Die Internationale Weltkarte $1: 1000000$ und ihr heutiger Stand. Mitteil. des Reichsamtes für Landesaufnahme. Berlin 1931/32, 6-22.

15. PENCK, A.: Etablissement et publication d'une carte de la terre au 1:1000000. 11. Jahresbericht der Geogr. Gesellschaft von Bern, 1891/92, 1-32.

16. - Die Weltkarte $1: 1000000$. Jahrb. der Kartographie 1, 1941, 81-82.

17. Pratt, R. R.: The millionth map of Hispanic America. Geographical Review 17, 1927, 301-?.

18. Sтоскs, Тн.: Die Darstellung des Meeres auf der Internationalen Weltkarte $1: 1000000$. Wissenschaftl. Veröffentlichungen des Deutschen Museums für Länderkunde. Leipzig N. F. Nr. 4, 1936. 


\title{
LA CARTE INTERNATIONALE DU MONDE 1:1000000
}

La carte internationale du monde au $1 / \mathrm{M}$ a été proposé par $\mathrm{A}$. PENCK à l'occasion du congrès internationale en 1891, à Berne. Après une période critique, la réalisation pris court en 1911. Les besoins militaires de la guerre de 1914 à 1918 lui donnèrent un grand essort. Après celle-ci, l'importance de cette carte se développe par les contributions d'environ 35 services cartographiques officielles et autres institutions. Durant la deuxième guerre mondiale, le développement reprit enormément par les travaux des grandes puissances belligerantes, c'est-à-dire la Russie, l'Allemagne, l'Italie et les Anglo-Saxons. L'auteur espère de la Suisse la présentation d'une feuille d'un travail fini, pouvant être présentée convenablement dans cet œuvre.

\section{LA CARTA INTERNAZIONALE DEL MONDO 1:1000000}

La carta internazionale del mondo venne proposta da A. PENCK al congresso geografico internazionale a Berna nel 1891. Superati gli stadi critici iniziali, il lavoro concreto ebbe inizio nel 1911. Impulso diedero necessità militari della prima guerra mondiale. Nel tempo successivo vi contribuirono 35 istituti cartografici ed altre istituzioni. Durante la seconda guerra mondiale furono enormi i contributi degli stati belligeranti (Russia, Germania, Italia e Stati Anglo-Sassoni). L'autore spera in un contributo della Svizzera di modo che essa possa essere rappresentata onorevolmente con un foglio di qualità in questa opera di si ampia portata.

\section{KOTENSTREUUNG UND RELIEFFAKTOR}

\author{
Von Fritz Gassmann und Heinrich Gutersohn
}

\section{Mit 19 Abbildungen}

\section{$\S 1$. EINLEITUNG}

Das Relief als Landschaftselement zahlenmäßig zu erfassen, wurde schon häufig versucht. Nach einer Zeit eingehender Pflege (PENCK ${ }^{1}$ ) traten morphometrische Untersuchungen wieder stark zurück. Es fehlte ihnen wohl vor allem die praktische Anwendung. Solange sie aber nur um ihrer selbst willen betrieben werden, können sie wenig befriedigen. Im Streben nach immer besserer Erkenntnis der Landschaft, nach Auswertung statistischer Angaben auch über Kulturlandschaften, die zu deren Vergleich und Klassierung leiten, wird indessen die Zahl als knappste Möglichkeit der Charakterisierung immer wieder heranzuziehen sein. Nur muß Klarheit darüber herrschen, was mit dieser Zahl erfaßt werden soll, unter welchen Einschränkungen sie zum Vergleich herangezogen werden darf, was sie anderseits nicht enthalten kann, und daß sie auf alle Fälle das Relief nicht eindeutig bestimmt. Die Kurvenkarte allein vermag die Oberflächenformen mit genügender Genauigkeit festzulegen; die gewonnene Zahl dagegen erhält ihren Wert erst beim Vergleich, wenn möglich in Relation mit weiteren Gegebenheiten.

Zu den häufig verwendeten Begriffen der Morphometrie gehört die Reliefenergie. Man versteht darunter die relative Höhe eines bestimmten Landschaftsausschnittes. Die Reliefenergie vermag gute Anhaltspunkte über die Oberflächengestalt jedes Ausschnittes der Lithosphäre zu geben. Sie.ist indessen nicht eindeutig definiert; denn im $\mathrm{Be}-$ streben, eine möglichst wertvolle $\mathrm{Zahl}$ zu geben und gleichzeitig subjektives Ermessen des Bearbeiters auszuschalten, wandelte sich dieser Begriff mehrfach.

PARTSCH ${ }^{2}$ bezog die Reliefenergie auf willkürlich gewählte Landschaftsausschnitte von je $32 \mathrm{~km}^{2}$ und definierte sie als Abstand zwischen dem Niveau der Höhenscheitel und dem der Talgründe. KREBS ${ }^{3}$ versteht darunter den Höhenunterschied zwischen

1 Penck, A.: Morphologie der Erdoberfläche, I, Stuttgart 1894.

2 Partsch, J.: Schlesien, eine Landeskunde für das deutsche Volk, II, S. 586, Breslau 1911.

${ }^{3}$ KrEBS, N.: Eine Karte der Reliefenergie Süddeutschlands. Petermanns Mitteil., 68, 1922, S. 49-53. 